tuberculosis. ${ }^{1}$ The inflammatory ocular patterns were: panuveitis (66), posterior uveitis (PU), ${ }^{31}$ intermediate uveitis (IU), ${ }^{5}$ anterior uveitis (AU), ${ }^{12} \mathrm{AU}$ and $\mathrm{PU},{ }^{2} \mathrm{AU}$ and IU, ${ }^{1} \mathrm{IU}$ and PU, ${ }^{1}$ exudative retinal detachment, ${ }^{3}$ vasculitis, ${ }^{4}$ pseudotumor, ${ }^{1}$ scleritis $^{2}$ and sclero-uveitis. ${ }^{1}$ Bilateral ocular involvement was observed in 74 cases. Patients were assessed at basal visit and day $2-5,7,15$ and 30 after IVMP. The main outcome variable was best corrected visual acuity (BCVA), the degree of inflammation of the anterior chamber and vitreous, and macular thickness (macular oedema defined by OCT $>300 \mu \mathrm{m}$ ). The results are expressed as mean $\pm S D$ for normally distributed variables, or as median [interquartile range] when are not. Comparison of continuous variables was performed using the Wilcoxon test.

Results: We studied 74 \&/55 3 ; mean age $42 \pm 14.3$ years. IVMP dose ranged from $40-1000 \mathrm{mg} /$ day for $3-5$ consecutive days. Prior to IVMP, cycloplegic and corticosteroid eye drops were used in all cases. Improvement was faster among patients with inflammation in anterior chamber and vitritis than in BCVA, CME, retinitis and retinal vasculitis (TABLE). Total remission was achieved in $19.4 \%$ of the 129 patients after IVMP. In 126 of the patients continued with oral corticosteroids and 4 received an intraocular dose of steroids. These immunosuppressive drugs were added: Methotrexate (52), Cyclosporine A, ${ }^{48}$ Azathioprine ${ }^{40}$ and others. ${ }^{5}$ In a few cases biological therapy (64) was administered afterwards. In general, acute respiratory infection was the side-effect most frequent.

Abstract SAT0622 - Table 1

\begin{tabular}{|c|c|c|c|c|c|}
\hline & \multirow{2}{*}{$\begin{array}{c}\text { Affected eyes } \\
\text { baseline }\end{array}$} & \multicolumn{4}{|c|}{ Affected eyes after high-dose IVMP (\%) } \\
\hline & & Day 2.3 & Day 7 & Day 15 & Day 30 \\
\hline BCVA, mean $\pm S D$ & $0.47 \pm 0.35$ & $0.56 \pm 0.34^{*}$ & $0.61 \pm 0.33^{*}$ & $0.68 \pm 0.31^{*}$ & $0.75 \pm 0.3^{*}$ \\
\hline \begin{tabular}{|l}
$\begin{array}{l}\text { Anterior chamber cells } \\
\text { [median (IQR)] }\end{array}$ \\
\end{tabular} & $0.75[0-4]$ & $0[0-4]^{*}$ & $0[0-4]^{*}$ & $0[0.3]^{*}$ & $0[0-2]^{*}$ \\
\hline Vitrntis [median (IQR)] & $0[0-5]$ & $0[0.5]^{*}$ & $0[0-5]^{*}$ & $0[0-3]^{*}$ & $0[0-3]^{*}$ \\
\hline $\mathrm{OCT}$ (microns) mean $\pm \mathrm{SD}$ & $405.36 \pm 189.1$ & $365.49 \pm 159.7^{\star}$ & $346.48 \pm 150.9^{*}$ & $308.2 \pm 120.06^{*}$ & $281.79 \pm 107.6^{*}$ \\
\hline $\begin{array}{l}\text { Choroiditis/Chorioretinitis n, } \\
(\%)\end{array}$ & $59(23 \%)$ & $51(20 \%)$ & $33(13 \%)$ & $23(9 \%)$ & $15(5 \%)$ \\
\hline Retinitis n, (\%) & $86(34 \%)$ & $74(29 \%)$ & $70(27 \%)$ & $46(18 \%)$ & $21(8 \%)$ \\
\hline Retinal vasculitis n, (\%) & $66(26 \%)$ & $58(23 \%)$ & $52(20 \%)$ & $36(14 \%)$ & $16(6 \%)$ \\
\hline Synechiae n. (\%) & $39(15 \%)$ & $32(12 \%)$ & $30(12 \%)$ & $30(12 \%)$ & $33(13 \%)$ \\
\hline $\begin{array}{l}\begin{array}{l}\text { Cystoid macular edema } \\
\text { (CME) n, (\%) }\end{array} \\
\end{array}$ & $83(33 \%)$ & $68(27 \%)$ & $57(22 \%)$ & $41(16 \%)$ & $20(8 \%)$ \\
\hline
\end{tabular}

${ }^{*} p<0.05$ compared with basal

Conclusions: High-dose IVMP pulse therapy is beneficial in the prompt control of severe uveitis and it is well tolerated.

Disclosure of Interest: None declared

DOI: 10.1136/annrheumdis-2018-eular.3780

\section{SAT0623 CLINICAL FEATURES AND TREATMENT OF IDIOPATHIC RECURRENT ACUTE PLEURO-PERICARDITIS}

K. Dietz, H.-M. Lorenz, N. Blank. Rheumatology, Internal Medicine 5, University Hospital Heidelberg, Heidelberg, Germany

Background: Idiopathic recurrent acute pleuro-pericarditis (IRAP) is an increasingly recognised autoinflammatory disease comprising post-pericardiotomy-syndrome, recurrent pericarditis and post-myocardial-infarction-syndrome. Different autoimmune mechanisms were discussed in the past. Recently, IRAP is considered as an autoinflammatory disease. Therapeutic options comprise colchicine, prednisolone and interleukin (IL)-1beta blockingagents.

Objectives: to investigate whether idiopathic and post-interventional pleuro-pericarditis represent a clinical spectrum and to identify treatment options.

Methods: This study analyses demographic, clinical and laboratory features of post-interventional and idiopathic pleuro-pericarditis and adult onset Still's disease $(A O S D)$ as a reference. Patients with infectious disease, connective tissue disease, chronic heart failure, renal failure and other non-exsudative effusions were excluded from this analysis. Patients with IRAP were treated with colchicine, prednisolone and IL1 $\beta$ blocking agents.

Results: Between 2005 and 201766 cases of idiopathic and post-interventional pleuro-pericarditis were identified and compared to 83 cases of AOSD. Clinical and laboratory features suggest that idiopathic and post-interventional pleuropericarditis represent a clinical spectrum which is identical with IRAP.

Prednisolone was started with $25 \mathrm{mg}$ to $125 \mathrm{mg}$ and tapered to less than $7 \mathrm{mg}$ or discontinued if not effective. 47 of 66 patients $(71 \%)$ were treated with prednisolone and $10 / 47(21 \%)$ were in remission with no need of any further therapeutically escalation.

Colchicine was given to $44 / 66$ patients (67\%) and $29 / 44$ (66\%) were in complete remission.

Four of 66 patients (6\%) did not respond or had contraindications against colchicine or prednisolone and were treated with anakinra. Of these patients $4 / 4(100 \%)$ were in remission. During the follow-up period of 20 patient ${ }^{*}$ months 2 of 4 patients maintained the remission with anakinra every 2 nd day and two patients discontinued anakinra and remained in remission.

Conclusions: 1. post-pericardectomy-syndrome, post-myocardial-infarctionsyndrome and idiopa-thic recurrent pericarditis represent a clinical spectrum of autoinflammatory diseases.

2. treatment options comprise colchicine as a first-line therapy, prednisolone and anti-IL1 blocking agents.

Acknowledgements: none.

Disclosure of Interest: None declared

DOI: 10.1136/annrheumdis-2018-eular.3027

\section{SAT0624 INCIDENCE AND BASELINE CHARACTERISTICS OF RELAPSE OR EXACERBATION IN PATIENTS WITH PULMONARY SARCOIDOSIS: A SINGLE CENTRE LONG- TERM OBSERVATIONAL COHORT STUDY}

O. Murata ${ }^{1,2}$, A. Kudo ${ }^{2}$, K. Suzuki ${ }^{3} .{ }^{1}$ Division of Pulmonary Medicine, Allergy, and Rheumatology, Department of Internal Medicine, School of Medicine, Iwate Medical University, Morioka; ${ }^{2}$ Department of Pulmonary Medicine, Hachinohe Red Cross Hospital, Hachinohe; ${ }^{3}$ Division of Rheumatology, Department of Internal Medicine, School of Medicine, Keio University, Tokyo, Japan

Background: Sarcoidosis is a systemic granulomatous disease that can affect multiple organs. Lung involvement is particularly common, occurring in more than $90 \%$ of patients. ${ }^{1}$ However, spontaneous remission of pulmonary sarcoidosis is often observed within 2 years. ${ }^{2}$ Factors reported to indicate a poor prognosis in pulmonary sarcoidosis include chest radiographic stage, skin involvement, age over 40 years and smoking history. ${ }^{3,4}$ To date, however, no study has comprehensively described factors of relapse and exacerbation and characteristics of untreated patients with favourable outcomes over a long-term period of more than 2 years.

Objectives: The aim of study was to identify the incidence and baseline characteristics of relapse and exacerbation in patients with pulmonary sarcoidosis over a long-term period.

Methods: One hundred- and three patients who visited our division of Hachinohe Red Cross Hospital between January 2007 and December 2016 and were clinically diagnosed as having pulmonary sarcoidosis were enrolled. The incidence and characteristics of relapse or exacerbation were prospectively calculated and statistically analysed using clinical, laboratory and imaging data collected from medical records.

Results: Of 103 patients, $79 \%$ were women and mean age at diagnosis was 50.1 \pm 16.4 years. Mean observation period was $9.8 \pm 8$. 6 years. The number of patients with previously reported short-term prognostic factors ware as follows: chest radiographic stage (Stage $0, n=7$; Stage $\mathrm{I}, \mathrm{n}=32$; Stage II, $\mathrm{n}=36$; Stage III, $\mathrm{n}=18$ Stage IV, $n=10)$, skin involvement $(n=9)$, age over 40 years old $(n=73)$, and smoking history $(n=38)$. Overall relapse or exacerbation was $22.3 \%(n=23)$ and mean period to relapse or exacerbation was $8.7 \pm 8.3$ years. We then analysed the 69 patients who were observed for more than 5 years, and identified relapse or exacerbation within 5 years in 9 patients. Comparison of characteristics at diagnosis between the relapse or exacerbation group and spontaneous group showed that the relapse or exarcerbation group had a significantly elevated frequency of bilateral hilar lymphadenopathy, disease duration, ocular involvement, cardiac involvement, and oral glucocorticoid use at diagnosis $(p=0.014,0.027,0.019$ 0.035 , and 0.0043 , respectively). On stratification, the number of risk factors was positively and significantly associated with the cumulative ratio of relapse or exacerbation $(p=0.048)$.

Conclusions: Our long-term observational cohort study newly identified the incidence and baseline characteristics of relapse or exacerbation in patients with pulmonary sarcoidosis over a long-term period. Scoring the number of the factors at baseline may facilitate the prediction of relapse or exacerbation.

\section{REFERENCES:}

[1] Br. J. Dis. Chest 1976;70:206-210.

[2] Dan. Bull. Med 1982;29:27-32.

[3] Br. Med. J 1961;2:1165-1172.

[4] Chest 2006;130:1851-6.

Disclosure of Interest: None declared

DOI: 10.1136/annrheumdis-2018-eular.2745 